\title{
L'herbe est toujours plus verte dans le pré du voisin
}

\section{Christoph Bosshard}

Dr med., vice-président de la FMH, membre du Comité central de la FMH, responsable du département Données, démographie et qualité / Académie suisse pour la qualité en médecine (ASQM)

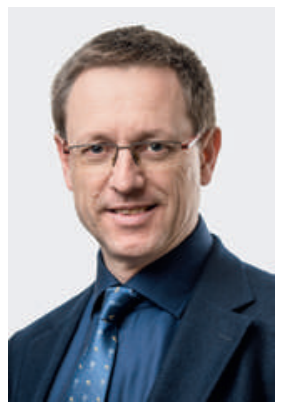

Il semble qu'il s'agisse d'un instinct inné: nous aimons la comparaison, à commencer par celle des images que nous renvoient les miroirs; ces images qui nous apprennent parfois quelque chose ou qui parfois ne font que confirmer ce que nous savons déjà. Grâce à l'International Health Policy Survey du Commonwealth Fund, à laquelle les médecins de famille suisses ont participé en 2015 pour la deuxième fois depuis 2012, nous disposons d'informations actuelles sur la situation des médecins de premier recours. Ces résultats étayent ce qui pour la FMH était, en partie déjà, évident au travers du traitement annuel des données statistiques: la dynamique de la démographie médicale dans notre pays révèle

Le phénomène de la démographie médicale: les fournisseurs de prestations sont de plus en plus souvent également les bénéficiaires des prestations.

une problématique particulière. En effet, les fournisseurs de prestations sont de plus en plus souvent également les bénéficiaires des prestations. La pyramide des âges des médecins de premier recours ne laisse entrevoir aucun espoir pour une relève suffisante. Les milieux médicaux lancent des appels dans ce sens depuis plus de dix ans, alors qu'à l'époque, notre société, et plus exactement les politiques, se penchaient encore sur l'hypothèse d'une suroffre médicale. La situation des médecins de premier recours est d'ailleurs la même que pour tous les autres spécialistes.

La FMH s'engage donc pour que la Suisse soit en mesure de couvrir elle-même ses besoins en médecins. L'importation actuellement nécessaire de praticiens étrangers nous place face à un problème moral, celui de la fuite des cerveaux. Cette situation ne pourra plus continuer ainsi à l'avenir. Dans le même temps, il s'agit de ne pas éloigner nos médecins des patients par une bureaucratie redondante ou inutile.

La hausse constante de la proportion de femmes interpelle également. Traditionnellement, les métiers masculins sont plutôt orientés vers le profit tandis que les professions féminines penchent davantage vers le social. Aujourd'hui, rares sont ceux qui contestent que la profession de médecin est devenue une profession féminine. Ce phénomène s'accompagne également de l'aug- mentation du travail à temps partiel, une tendance qui n'est assurément pas qu'une seule question de genre. En revanche, c'est un défi à prendre en compte si nous voulons discuter de la prise en charge médicale de demain.

Ce n'est pas sans sourire que j'ai pris note des déclarations de l'étude concernant l'excellent niveau de mise en réseau d'une part et le faible niveau d'informatisation d'autre part. Une corrélation qui pourrait naturellement s'expliquer par la pyramide des âges mais qui rappelle cependant de manière irréfutable que le réseau n'est pas seulement constitué de structures et de processus mais qu'il est bien l'élément central de la connaissance mutuelle et de la confiance. Ce principe fondamental s'applique manifestement aussi bien à la relation médecin-patient qu'aux relations au sein du corps médical. Dans ce même numéro du Bulletin des médecins suisses, vous en apprendrez davantage sur l'International Health Policy Survey 2015 à la page 789.

En comparaison internationale, notre système de santé fait très bonne figure dans de nombreux domaines, et ceci également en ce qui concerne la bonne et rapide disponibilité des médecins. Notre système a, d'ailleurs, largement et plusieurs fois été plébiscité par le peuple suisse dans les urnes. Si les prestations médicales inu-

Le travail à temps partiel n'est pas qu'une seule question de genre et il faut en tenir compte pour la prise en charge médicale de demain.

tiles constituent aujourd'hui un défi, il faut surtout les considérer comme un effet collatéral du système. La FMH prend cette question très au sérieux, comme elle le souligne dans son document-cadre La surconsommation de prestations médicales: un problème de qualité [1]. Elle s'est également prononcée sur la décision partagée (shared decision making) et la qualité de l'indication. Pour nous, il est évident que rester bons signifie s'améliorer constamment. Mais là aussi, il faut tenir compte des principes d'efficacité, d'adéquation et d'économicité. Dès lors, la question se pose de savoir jusqu'où sommesnous prêts à aller et avec quels moyens? Ces discussions doivent se dérouler en toute objectivité. C'est le seul moyen de transmettre la fascination de notre profession aux jeunes générations, et de les motiver à collaborer. 